\title{
Soft X-Ray Techniques to Study Mesoscale Magnetism
}

\author{
Jeffrey B. Kortright \\ Lawrence Berkeley National Laboratory \\ Berkeley, California USA
}

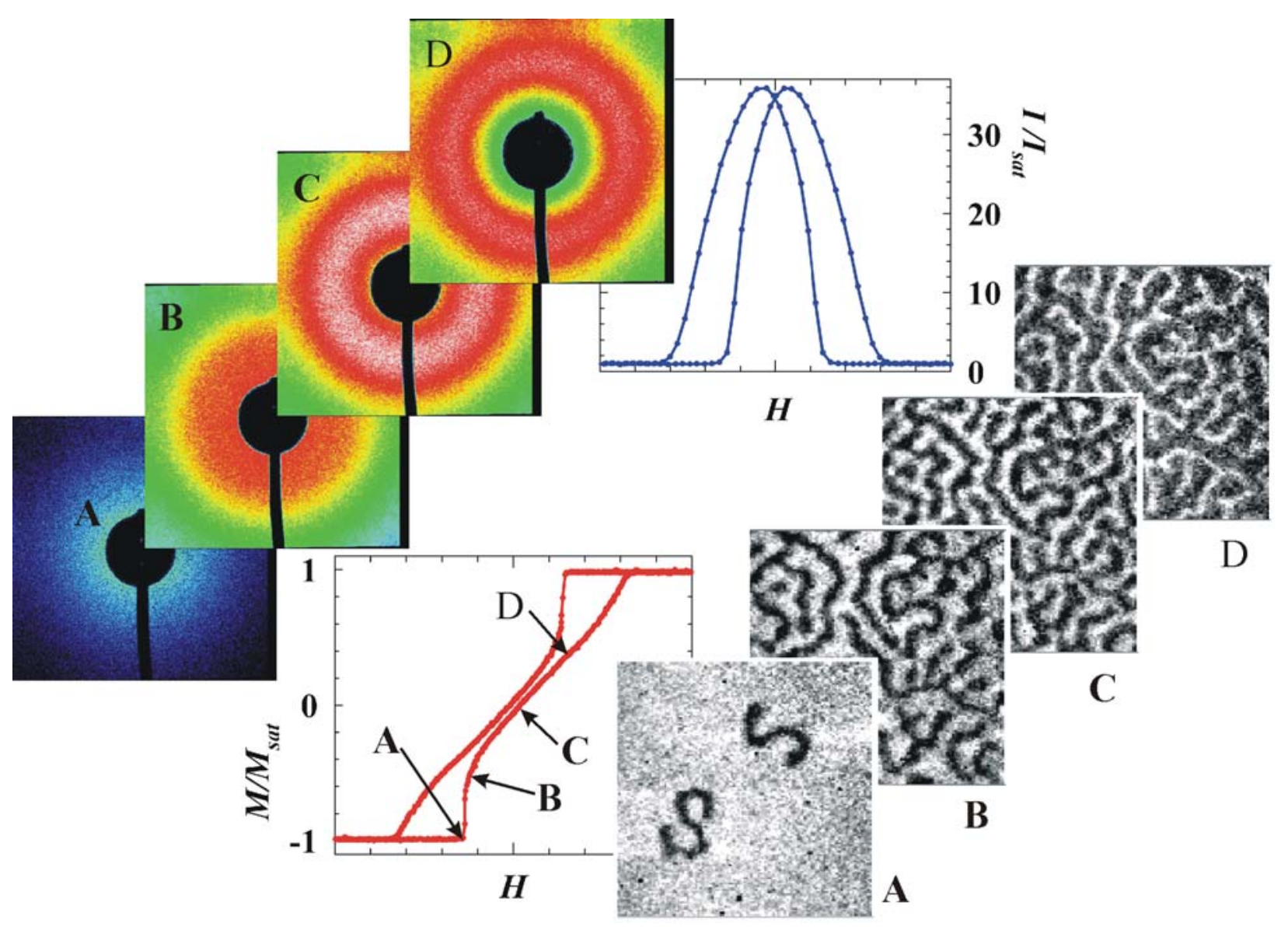

Cover Illustration. Different representations of magnetization (M) reversal in Co/Pt multilayers are shown on the cover. The plots are (red) a visible magneto-optic Kerr effect hysteresis loop showing the average, macroscopic $\mathrm{M}$ and (blue) a partially coherent $\mathrm{x}$-ray scattering loop resulting from deviations from the average $M$ at a fixed spatial frequency. The domains responsible for the scattering are seen directly in a sequence of $\mathrm{x}$-ray zone-plate microscope images 4.5 microns across (right). The corresponding sequence of fully coherent x-ray scattering patterns (left) contains the same information as the images encoded in the speckled intensity distrubutions. (Unpublished domain images and speckle patterns from similar samples courtesy O. Hellwig, S. Kevan, and L. Sorensen.) 


\title{
Soft X-Ray Techniques to Study Mesoscale Magnetism
}

\author{
Jeffrey B. Kortright \\ Lawrence Berkeley National Laboratory \\ Berkeley, California USA
}

Heterogeneity in magnetization (M) is ubiquitous in modern systems. Even in nominally homogeneous materials, domains or pinning centers typically mediate magnetization reversal. Fundamental lengths determining M structure include the domain wall width and the exchange stiffness length, typically in the $4-400 \mathrm{~nm}$ range. Chemical heterogeneity (phase separation, polycrystalline microstructure, lithographic or other patterning, etc.) with length scales from nanometers to microns is often introduced to influence magnetic properties. With 1-2 nm wavelengths $\lambda$, soft $x$-rays in principle can resolve structure down to $\lambda / 2$, and are well suited to study these mesoscopic length scales $[1,2]$. This article highlights recent advances in resonant soft x-ray methods to resolve lateral magnetic structure [3], and discusses some of their relative merits and limitations. Only techniques detecting x-ray photons (rather than photo-electrons) are considered [4], since they are compatible with strong applied fields to probe relatively deeply into samples.

The magneto-optical (MO) effects discovered by Faraday and Kerr were observed in the x-ray range over a century later, first at "hard" wavelengths in diffraction experiments probing interatomic magnetic structure [5]. In the soft x-ray range, magnetic linear [6] and circular [7] dichroism spectroscopies first developed that average over lateral magnetic structure. These large resonant MO effects enable different approaches to study magnetic structure or heterogeneity that can be categorized as microscopy or scattering [1]. Direct images of magnetic 
structure result from photo-emission electron microscopes $[4,8]$ and zone-plate microscopes $[9$, 10]. Scattering techniques extended into the soft x-ray include familiar specular reflection that laterally averages over structure but can provide depth-resolved information, and diffuse scattering and diffraction that provide direct information about lateral magnetic structure. Scattering techniques are further classified as partially for fully coherent according to the extent of transverse coherence of the incident beam.

The techniques utilize the resonant atomic scattering factor [11] $f=\left(\mathbf{e}_{\mathbf{f}}^{*} \cdot \mathbf{e}_{\mathbf{o}}\right) f_{c}+i\left(\mathbf{e}_{\mathbf{f}}^{*} \times \mathbf{e}_{\mathbf{o}}\right) \cdot \mathbf{m} f_{m 1}+\left(\mathbf{e}_{\mathbf{f}}^{*} \cdot \mathbf{m}\right)\left(\mathbf{e}_{\mathbf{o}} \cdot \mathbf{m}\right) f_{m 2}$ where $\mathbf{e}_{\mathbf{o}}$ and $\mathbf{e}_{\mathbf{f}}$ are polarization vectors of incident and scattered $\mathbf{x}$-rays and $\mathbf{m}$ is a unit vector in the direction of the local magnetization. The absorptive parts of the first- $\left(f_{m 1}\right)$ and second- $\left(f_{m 2}\right)$ order magnetic terms represent magnetic circular and linear dichroism (MCD and MLD), respectively. Primarily the charge term $\left(f_{c}\right)$ and $f_{m 1}$ are considered here, since $f_{m 2}$ is small compared to $f_{m 1}$ for many samples [12]. $f_{c}$ and $f_{m 1}$ for Fe across its $L_{2,3}$ edges are seen in Figure 1. Compared to hard x-rays, stronger soft x-ray absorption reflects a larger interaction cross-section that provides high sensitivity to small sample volumes, and limits studies to thin films or near surface regions of bulk samples [12].

Early soft x-ray scattering studies used the off-specular reflection geometry to resolve magnetic and chemical roughness at surfaces $[13,14]$. Such studies initially used circular incident polarization, and measured off-specular scattering either in [14], or out of [13, 15], the plane of specular reflection. Intensities $I_{+}$and $I_{-}$collected with opposite helicity yield $I_{+}-I_{-}$, that was initially interpreted as giving magnetic intensity, and $\left(I_{+}+I_{-}\right) / 2$ as the charge (or chemical) scattering. Differences in the angular profiles of these quantities were interpreted to reflect different power spectra of interfacial magnetic and chemical roughness. These studies 
found that the difference falls off faster than the sum with in-plane scattering vector $\boldsymbol{q}$, and concluded that magnetic roughness has longer in-plane correlation lengths than chemical roughness. Studies have since pointed out that $I_{+}-I_{-}$corresponds to the charge-magnetic interference term [16], and that angle-dependent optical and MO effects can complicate measurement of the shape of the different structural correlation functions of interest [12].

Different types of magnetic and chemical heterogeneity have been studied with resonant scattering. This heterogeneity is distinct from topological surface roughness, and may take the form of magnetic domains or grains in polycrystalline films. Films with perpendicular anisotropy exhibit up and down stripe domains, illustrated in Fig. 2, and form an important class of samples in which scattering from domains has received extensive early study $[17,18]$. Systems with relatively weak anisotropy tend to form surface closure domains, as in Fig. 2a, that become difficult to study as the domain widths decrease. An early study of FePt films with weak anisotropy revealed that the periodic domain structure forms a magnetic grating that scatters into a series of diffraction orders [17]. The $+/$ - asymmetry of these peaks with helicity reversal is observed only in a specific geometry [19], indicating that in-plane M components are located at perpendicular domain boundaries as in Fig. 2a. The incidence angle dependence of the asymmetry provides a measure of the depth of the surface closure domains.

Other studies have utilized symmetric transmission geometry familiar from small-angle scattering [18]. Transmission geometry requires semitransparent samples that are consistent with many thin film systems of interest (typically grown on $100-150 \mathrm{~nm}$ SiNx membranes), and positions $\boldsymbol{q}$ in the sample plane to optimize its coupling to in-plane structure. These studies have primarily utilized linear incident polarization equivalently described as a coherent superposition of opposite helicity circular components. The intensity scattered by linear polarization, 
$I_{\text {lin }}=I_{+}+I_{-}$, is the sum of the scattering of the orthogonal circular components. Taking the scattering amplitude for + and - helicity components as $a_{+/-}=f_{c} \pm i f_{m 1}$, we find $I_{+}+I_{-} \propto 2 f_{c}^{*} f_{c} S_{c-c}+2 f_{m 1}^{*} f_{m 1} S_{m 1-m 1}$ where partial structure factors $S_{c-c}$ and $S_{m 1-m 1}$ give the autocorrelation of charge and magnetic structure. This expression assumes forward scattering and that $\mathbf{m}$ is oriented only parallel or anti-parallel to the beam. Linear polarization thus yields scattering from charge-charge and magnetic-magnetic correlations, but not from the chargemagnetic cross term.

Transmission scattering studies of $\mathrm{Co} / \mathrm{Pt}$ multilayers reveal both of these contributions [18], as seen in Figure 3 that shows data as a function of $q$ and $h v$ both at saturation (1.1 Tesla) and at $0.1 \mathrm{~T}$. A peak at $q \cong 0.0042 \AA^{-1}(2 \pi / q=150 \mathrm{~nm}$ spacing $)$ disappears when the sample is saturated, leaving a weaker peak at $q \cong 0.031 \AA^{-1}$ (20 nm spacing). The field dependence indicates that the low $q$ peak results from the labyrinth (disordered) stripe domains, and the high $q$ peak from non-magnetic (charge) structure. Confirmation of the origins of the two peaks comes from two distinct sources. One source is direct imaging. Domain images were obtained with transmission x-ray (see cover) and magnetic force microscopy; the domain power spectra match the position and shape of the low $q$ peak in Fig. 3a. Atomic force microscopy images surface topography associated with the polycrystalline grain structure, whose power spectra match the position and shape of the high $q$ peak. The second source is the very different Co $L_{2,3}$ energy spectra at the two peaks (Fig. 3b). Using measured values of Co $f_{c}$ and $f_{m 1}$, the only consistent model spectra are those assuming pure magnetic-magnetic intensity at the low $q$ peak, and pure charge-charge intensity at the high $q$ peak [18]. Modeling of energy spectra at different peaks in $q$ space has also been applied to resolve the origin of magnetic and chemical peaks in 
granular recording media [20], and appears to be valuable both to distinguish different sources of scattering and to quantify their chemical and magnetic makeup.

$\mathrm{Co} / \mathrm{Pt}$ multilayers and similar films provide model systems to study $\mathrm{M}$ structure in films whose energetics are altered by introducing perpendicular exchange-bias [21], antiferromagnetic coupling [22], microstructural disorder [23], lithographic patterning [24], and domain order [25]. Resonant scattering has been used to follow the effects of these interactions on domain structure and reversal behavior. Such modifications may be important in future generations of magnetic recording or other technology.

The above discussion raises questions concerning the utility of scattering vis-à-vis microscopy to study magnetic heterogeneity since, after all, microscopy was used to image the same domains that scatter at low $q$ (see cover and Fig. 3). Before comparing microscopy with scattering, the status of soft x-ray zone-plate magnetic microscopy is briefly reviewed [4].

Two distinct types of transmission zone-plate microscopes, imaging and scanning, have been utilized to image magnetic structure. An early imaging microscope at BESSY provided the first images of magnetic structure, in the form of stripe domains in $\mathrm{Gd} / \mathrm{Fe}$ multilayers having perpendicular anisotropy [9], much like those on the cover. Such studies are currently conducted at the ALS [26], and have expanded to include patterned films with perpendicular anisotropy [27, 28], field-dependent domain behavior [29, 25], and extended [30] and patterned films having inplane anisotropy.

The scanning transmission X-ray microscope (STXM) at the ALS [31] produced the first magnetic images in scanning mode, of domain and domain-boundary structures in demagnetized films having in-plane anisotropy $[10,32]$. Tilting is required to obtain MCD contrast for inplane $\mathrm{M}$, and multiple angle imaging of the same region was used to reconstruct the vector 
nature of the magnetization in a region where one domain is growing into another as shown in Figure 4. The large image shows in-plane M components, while the inset shows out-of-plane component [32]. A rich variety of structure is observed at and near the grain boundary, including hybrid Néel and Bloch domain walls, pronounced perpendicular magnetization components at vortex cores, and vortex pairs extending well away from the main domain wall. While these structures were known to exist before this study, the improved spatial resolution coupled with quantitative vector imaging capabilities are expected to yield new information on a range of magnetic features as they become more routinely used.

Currently two soft x-ray zone-plate microscopes are in use for magnetic studies (both at the ALS); the imaging instrument [26] on a bending magnet and an updated STXM recently moved to an elliptically polarizing undulator beamline. Both operate near the zone-plate diffraction limit, providing roughly $30 \mathrm{~nm}$ spatial resolution. Both are designed for transmission, and while neither was designed for magnetic studies, each is equipped with sample tilt capability and an electromagnet providing fields up to several kOe. Dynamic studies using pump-probe techniques are compatible with both microscopes [33]. The polarization control and spectral resolution of each microscope are quite different, which together with their different imaging modes makes them complementary in many respects. New zone-plate microscopes dedicated to magnetic studies could add significantly to the capabilities of existing instruments.

Microscopy and scattering have complementary sensitivity to magnetic heterogeneity as seen on the cover. Images provide direct, detailed information about local magnetic structure, especially isolated or individual features, unavailable from scattering. The correlation functions from scattering, on the other hand, typically average over a larger ensemble than do images, providing valuable statistical information. In addition to these well-known attributes, other 
differences are emerging as relevant in their comparative capabilities. Spatial resolution in microscopy scales inversely with zone-plate numerical aperture $\mathrm{NA} \cong \lambda / \Lambda$ and is limited by the outer zone width $\Lambda$ to $\sim 20-30 \mathrm{~nm}$. Scattering can be measured out to backscattering, with an effective NA $(\propto q)$ of 1 , and so can resolve features ten times smaller than microscopy. This larger spatial frequency range over which signals are inherently Fourier filtered, coupled with a larger dynamic range, gives scattering significantly greater sensitivity to mixed heterogeneity than microscopy. For example, simultaneous sensitivity to magnetic and chemical structure below the zone-plate resolution limit has been obtained in scattering measurements of granular recording media $[20,34,35]$. The more open geometry of scattering experiments may make them more amenable to utilization in a variety of sample environments.

Emerging fully coherent magnetic scattering provides three distinct approaches to gain additional information compared to the partially coherent scattering techniques discussed above. Each makes use of the speckled distribution of scattered intensity (see cover) that encodes the full spatial details of the magnetization distribution. Because coherent flux scales as $\lambda^{2}$ for a given brightness, the soft x-ray spectral range is attractive to utilize these techniques [36] and magnetic speckle originating from $f_{m 2}$ [37] as well as $f_{m 1}[38,39,23]$ has been observed. One approach is a lensless form of microscopy in which the speckle is inverted to obtain a real space image [40]. Inversion studies of magnetic speckle have been carried out using real [41] and simulated data $[41,42]$. While promising, concerns about the uniqueness of resulting images are not currently resolved. The second approach is the analog of dynamic light scattering, or time correlation spectroscopy, initially developed using lasers. Here temporal intensity fluctuations in a single speckle provide information about sample dynamics at that spatial frequency. This approach has been applied in the soft x-ray to study dynamic, non-resonant, charge scattering 
down to microsecond time resolution in liquid crystals [36]. Studies of magnetization dynamics with similar or possibly better time resolution should be feasible.

The third approach uses changes in the magnetic speckle with applied field to follow changes in domain structure $[43,23]$. This speckle metrology measures local domain correlations by cross-correlating their speckle patterns in $q$-space at different fields. Applied to study microscopic return-point memory in $\mathrm{Co} / \mathrm{Pt}$ multilayers having relatively little structural disorder, the cross correlations indicate that no memory exists between domains that form in successive excursions around the hysteresis loop. When microstructural disorder is significant however, the cross-correlations retain a similar, large value indicating pronounced microscopic domain memory over repeated hysteresis cycles [23]. Compared to speckle inversion, this approach sidesteps the uniqueness question to obtain statistical microscopic correlations. At the time of the measurements in Ref. 23, no soft x-ray microscopes had fields high enough to saturate the samples of interest.

In summary, the large, resonant, soft x-ray MO effects are being utilized to extend a variety of scattering and imaging techniques into this region. Both nanoscale lateral resolution and elemental specificity make these techniques directly relevant to study many magnetic systems of fundamental and technological interest. The real and reciprocal space techniques are truly complementary, and together provide more information either approach in isolation. Both microscopy and scattering offer potential for dynamic studies (driven and spontaneous) of nanoscale magnetism. As these techniques and their required instrumentation become more established they will continue to contribute significantly to our understanding of magnetic systems that will be increasingly important as the era of nanoscience and technology unfolds. 
The author is pleased to acknowledge many useful interactions with collaborators E. E.

Fullerton, O. Hellwig, Sang-Koog Kim, S. D. Kevan, and L. B. Sorenson, M. Pierce, G. P.

Denbeaux, K. Chesnel, and T. Warwick and support from the Office of Science, Basic Energy

Sciences, U.S. Department of Energy under Contract No. DE-AC03-76SF00098.

\section{References}

1. J. B. Kortright, et al., J. Magn. Magn. Mater. 207, 7 (1999).

2. G. van der Laan, Synchrotron Radiation News, 14(5), 32 (2001).

3. For brevity discussion is limited to lateral magnetic structure. Soft x-rays are also used to depth-resolve magnetic and chemical structure, within skin-depth limitations.

4. Not discussed here is surface-sensitive photo-electron imaging microscopy that has provided much insight into exchange-bias and other systems. A recent review of PEEM studies of surface magnetism is: C. M. Schneider and G. Schonhense, Reports on Progress in Physics 65, R1785 (2002).

5. For a review of hard x-ray magnetic scattering see articles in Synchrotron Radiation News, 14(5), (2003).

6. B. T. Thole, et al., Phys. Rev. Lett. 55, 2086 (1985).

7. C. T. Chen, et al., Phys. Rev. B 42, 7262 (1990).

8. J. Stöhr, et al., Surf. Rev. Lett. 5, 1297 (1998).

9. P. Fischer, et al., J. de Physique IV 7-C2, 467 (1997); P. Fischer, et al., J. Phys. D: Appl. Phys. 31, 649 (1998).

10. J. B. Kortright, et al., J. Magn. Magn. Materials 191, 79 (1999).

11. J. P. Hannon, et al., Phys. Rev. Lett. 61, 1245 (1988); 62, 2644(E) (1989).

12. J. B. Kortright, and S.-K. Kim, Phys. Rev. B 62, 12216 (2000).

13. J. F. MacKay, et al., Phys. Rev. Lett. 77, 3925 (1996).

14. J. W. Freeland, et al., J. Appl. Phys. 83, 6290 (1998).

15. J. J. Kelly IV, et al., J. Appl. Phys., 91, 9978 (2002).

16. R. M. Osgood III, et al., J. Magn. Magn. Mater. 198-199, 698 (1999).

17. H. A. Dürr, et al.amson, Science 284, 2166 (1999). 
18. J. B. Kortright, et al., Phys. Rev. B 64, 092401 (2001).

19. E. Dudzik, et al., J. Appl. Phys. 87, 5469 (2000).

20. J. B. Kortright, et al., J. Magn. and Magn. Mater. 240, 325 (2002).

21. O. Hellwig, et al., Phys. Rev. B 65, 144418 (2002).

22. O. Hellwig, et al., Nature Materials, 2, 112-116 (February 2003).

23. M. S. Pierce, et al., Phys. Rev. Lett. 90, 175502 (2003).

24. K. Chesnel, et al., Phys. Rev. B 66, 2404 (2002).

25. O. Hellwig, et al., Physica B 336, 136 (2003).

26. G. Denbeaux, et al., IEEE Trans. Magn. 37, 2764 (2001).

27. T. Eimüller, et al., J. Appl Phys. 89, 7162 (2001).

28. G. J. Kusinski, et al., Appl. Phys. Lett. 79, 2211 (2001).

29. P. Fischer, et al., IEEE Trans. Magn., 38, 2427 (2002).

30. P. Fischer, et al., J. Appl. Phys. 89, 7159 (2001).

31. T. Warwick, et al., Journal of Synchrotron Radiation 5, 1090, (1998).

32. S.-K. Kim, et al., Appl. Phys. Lett. 78, 2724 (2001).

33. P. Fischer (private communication).

34. O. Hellwig, et al., Appl. Phys. Lett. 80, 1234 (2002).

35. E. E. Fullerton, et al., IEEE Trans. Magn. 38, 1693 (2002).

36. A. C. Price, et al., Phys. Rev. Lett. 82, 755 (1999).

37. A. Rahmim, et al., Phys. Rev. B 65, 235432 (2002).

38. F. Yakhou, et al., J. Magn. Magn. Mater. 233, 119 (2001).

39. K. Chesnel, et al., Pys. Rev. B 66, 172404 (2002).

40. J. Miao, et al., Nature 400, 342 (1999).

41. A. Rahmim, M.Sc. Thesis, University of British Columbia (1999).

42. T. O. Menteş, et al., J. Synchrotron Radiation 9, 90 (2002).

43. B. Hu, et al., Synchrotron Radiation News 14(2), 11 (2001). 

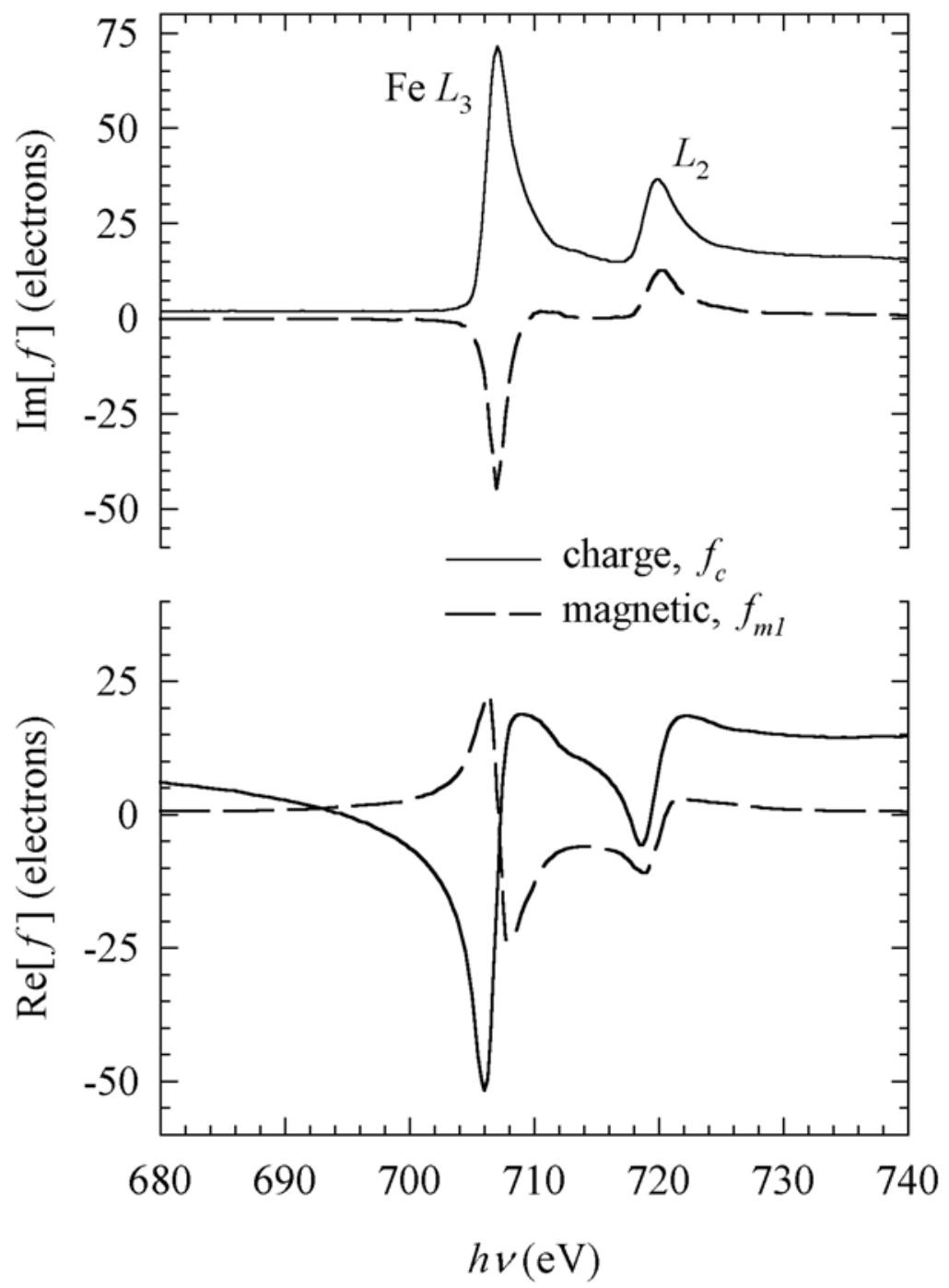

Figure 1. Measured charge and first-order magnetic atomic scattering factors for bulk Fe across its $L_{2,3}$ edges. (From Ref. [12].) 


\section{(A)}

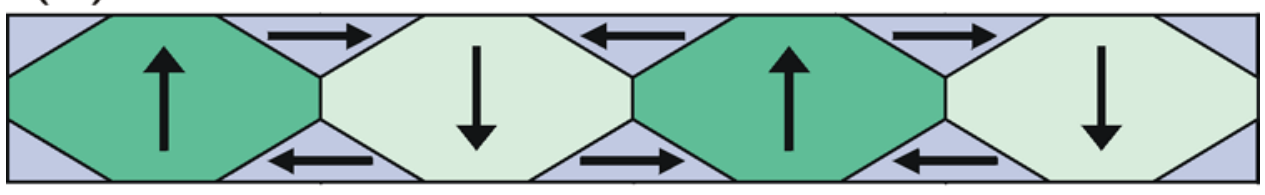

\section{(B)}

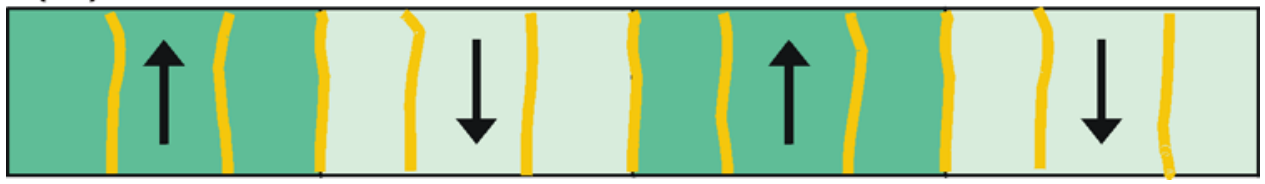

Figure 2. Stripe domains in thin films with perpendicular magnetic anisotropy may exhibit surface closure domains when anisotropy is relatively weak (a), while films with strong anisotropy may not (b). Perpendicular domains have typical lateral dimension $\sim 90-300 \mathrm{~nm}$, and are well suited for soft x-ray studies. Irregular lines in (b) indicate possible chemical heterogeneity (grain boundaries, segregation, etc.) that may be present in such films. 

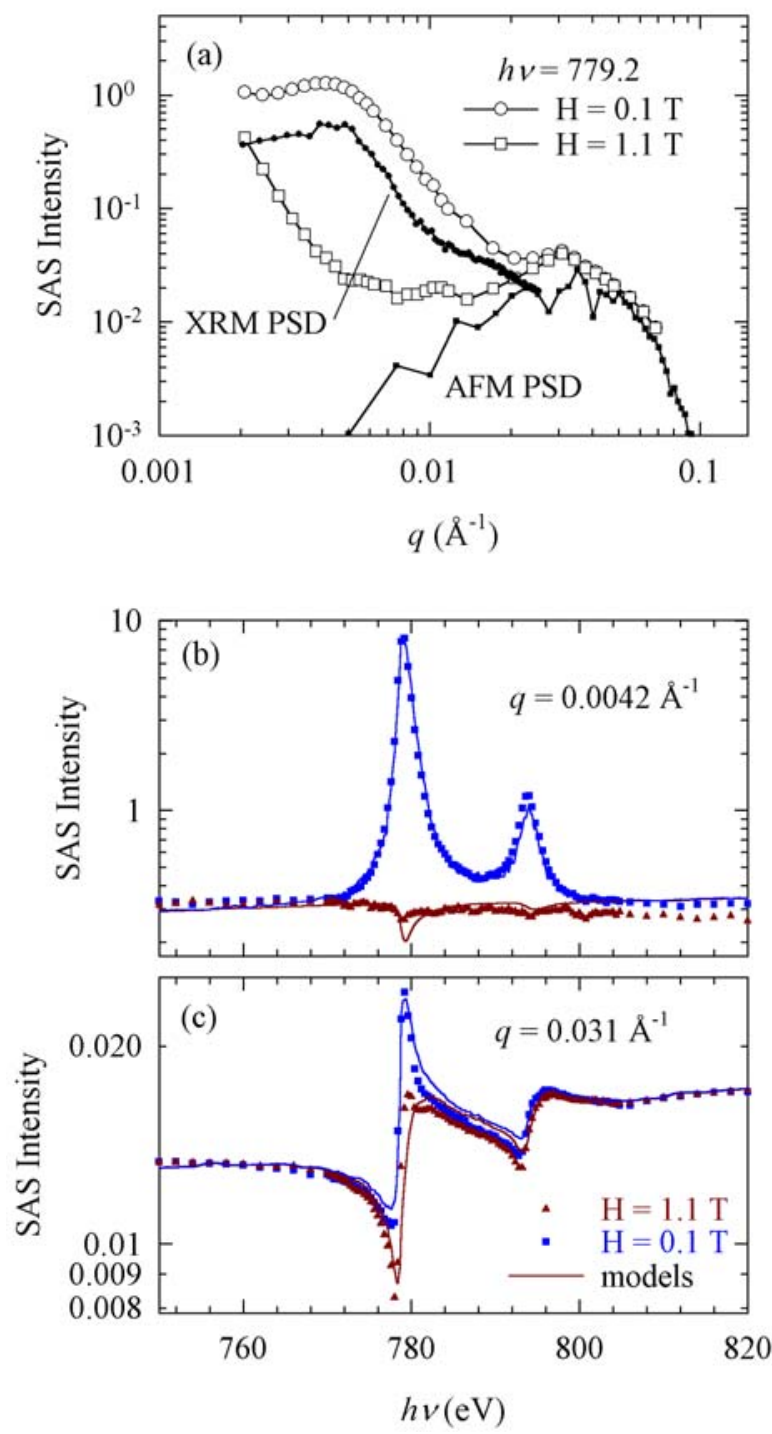

Figure 3. (a) Resonant scattering from a $\mathrm{Co} / \mathrm{Pt}$ multilayer using linear polarization shows two distinct peaks (open symbols) that correspond to magnetic and chemical correlation lengths. The low- $q$ magnetic peak disappears on saturation. The power spectral density (PSD) obtained from magnetic x-ray microscopy (MFM) and atomic force microscopy (AFM) confirm the identification of the magnetic and charge peaks. The energy spectra of the two peaks, (b) and (c), are modeled using measured charge and magnetic scattering factors to further confirm their origin. (From Ref. [18].) 


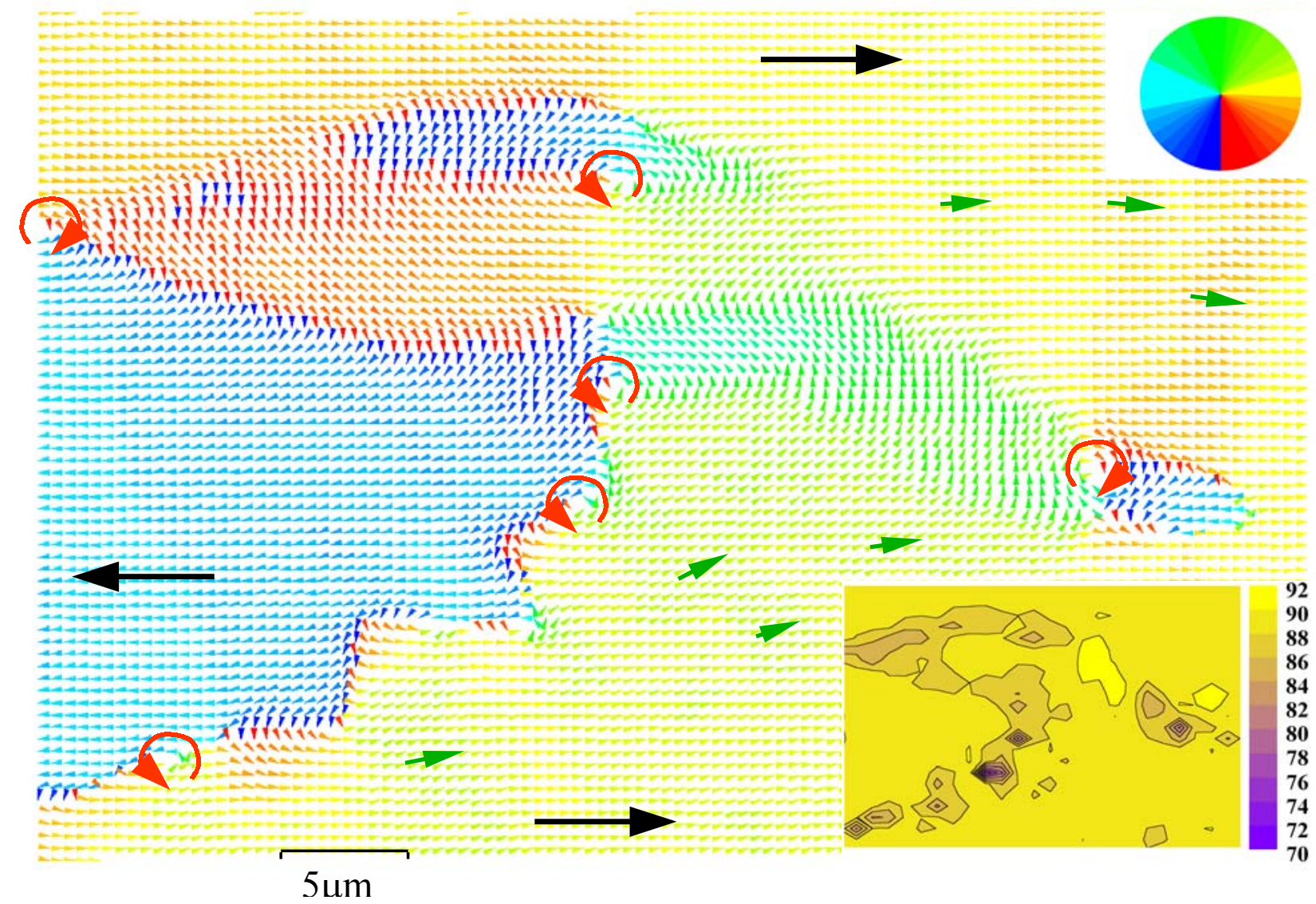

Figure 4. Scanning transmission microscope images of vector magnetization in the region where one domain (blue) is growing into another domain (yellow) with oppositely oriented magnetization in a demagnetized Fe film. The main image shows the in-plane magnetization components with color-coded directions over a 35 by $50 \mu^{2}$ field. The inset shows the out-ofplane components over the same field, with color scale indicating the angle out-of-plane $\left(90^{\circ}\right.$ corresponds to in-plane M). (From Ref. [10].) 